\title{
Design and Implementation of Wechat Marketing System on Web Mode
}

\author{
Guangyu Peng \\ Department of Mechanical and Electrical Engineering, Hunan Communication Polytechnic, \\ Changsha 410132, China; \\ paper@163.com
}

Keywords: Internet, wechat, marketing system, web mode.

\begin{abstract}
The recent fast development of WeChat platform gradually raises a new requirement of connecting abstract mobile internet with the physical world. This requires new protocols, new theories and also engineering practices that model WeChat platform and physical resources in a unified framework, a challenge that few current approaches are able to tackle, a new field that attracts lots of researchers devote themselves to exploring. The solution must break the boundary between the mobile internet world and the physical world by providing a unified infrastructure that permits integrated models addressing issues from both worlds simultaneously. This paper proposes a framework to integrate WeChat platform. A case study is presented to demonstrate the advantage of the framework.
\end{abstract}

\section{Introduction}

Under the conditions of the rapid development of mobile Internet, the $3 G$ era makes rapid popularity of smart phones, people would rather spend times on smart phones than PC, and with the promotion of smart phones from high-end market to the low-end market, the world's smart phone sales have reached 1.4 billion [1]. For a time, smart mobile terminals to support increased demand for mobile IM software, Which provides a good opportunity to IM software developers, that mobile IM software is not limited to meet the daily needs of the people, and also to constantly adapt to new media changes in the environment in order to constantly improve the user consolidate their place in the mobile terminal of the page [2]. Micro-channel mobile IM software as a rising star, with lightning speed mobile IM software charts quickly occupied the throne, has over 600 million users become social media, increasing its market demand, nearly 90 million businesses, wait public access to personal micro-channel platform micro-channel has become the largest mobile Internet portal .In this paper, micro letter to the object, try to micro-channel status of the case study analyzes the status quo micro-channel development and the reasons they are in hot pursuit in a short time, situation of enterprises in the current use of micro letter to do the marketing and the future how you can make use of the micro-channel marketing has made some explanation [3]. This paper hopes to explore the phenomenon through micro-channel direction and trend of future development, making the prospects for micro-channel accurately and efficiently estimate to enrich people's awareness and micro-channel enterprises and individuals to make use of a micro-channel marketing draw.

With the rapid development of economic society in China and guidance of national policy, popularizing rate of Internet has increased year by year. How to conduct network marketing effectively has become a topic in front of each enterprise. In China, there are numerous small and micro businesses. However, living environment of most small and micro businesses is very complicated, and they have many problems, such as shortage of funds, deficiency of talents, and lack of policy support. Network marketing is different from traditional marketing in marketing concept and actual operational approach. Network marketing can assist small and micro businesses in reducing marketing costs and breaking through limits of time and space. The difference between small and micro businesses and large and medium-size enterprises can be shortened through taking advantage of network marketing, so as to form their own advantages gradually on Internet platform.

Compared with traditional Internet, mobile Internet is faster in development and larger in market potential. Mobile network marketing will be the trend and direction in the future. In this paper, 
advantages and disadvantages of micro-bog marketing and micro-letter marketing, existing problems, possible development direction in the future, and Taobao marketing were introduced simply.

\section{Wechat public platform}

WeChat is a product of mobile internet provides instant messaging (IM) for the mobile client and the internet sever. It is a platform integrates text message communicate, voice messaging, broadcast (on-to-many), picture or location sharing and the exchange of contact information subtly [4]. Until now, WeChat have had the number of user more than 300 million users, indicating that it's a successful social Via location-based functions like 'Shake', 'Look Around', 'Drift Bottle' and so on WeChat can easily get user's information and know what they may need, and profit from the internet and the cloud technical, this requirement, if is indeed, can be instantaneously sent to every corner of the world. Besides, WeChat platform also provide an opened, convenient entrance for all users, which means every individual can have their own WeChat account on the platform whenever they want. By linking amount of all these users, WeChat platform construct a large network of social communication, actually this is the reason why we choose WeChat platform as the important node of our framework of wechat as well as the essential instrument to get the request and reply from the user. The dataflow in the system is shown in Fig.1.

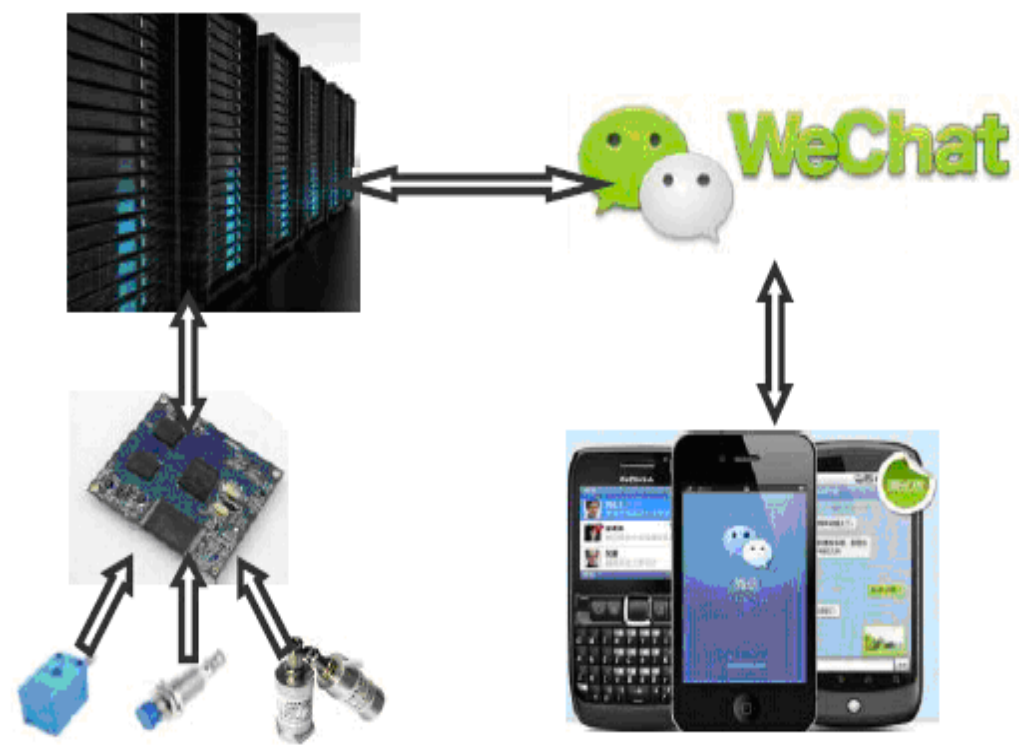

Fig. 1 The dataflow in the system

\section{Embedded system}

The Embedded System is supposed to illustrate almost everything have the ability of network wherever they are (primarily the sensors, actuators, etc.). It takes the responsibility of smoothing as well as package the data process. For the device which has low requirement of real-time, such as temperature and humidity sensors, the system should cache the data first and then send it block by block for the purpose of saving the bandwidth and also alleviate the servers' burden. Single chips can be mainly divided into two types, the fixed and the mobile. The former one is set in a fixed position and should register their location information in the database but the latter one can get their position through GPRG or GPS and then package the relevant data. Data package is shown in Fig. 2.

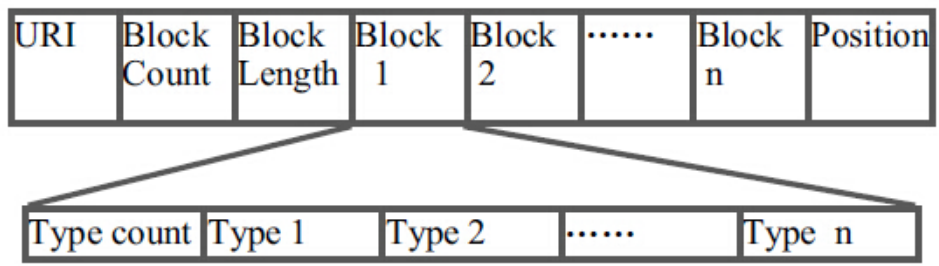

Fig. 2 Data package 
The URI is used for targeting. Servers distinguish the devices through the URI, ensuring that it can treat the data correctly and differently. Number of blocks is contained in the "Block Count" part and "Block Length" points out the length of each block. Followed by "Block Length", there are n blocks illustrating the message about terminal position. What's more, for each block, there may be more than one data being encapsulated into the package, just like DHT as an example, it encapsulates temperature and humidity data simultaneously. In this way, we can unify different kind of sensors together, which brings a great benefit to the developers. To enable networking, the embedded system should has the compatibility of GPRS, WIFI and also wire cable as well, but GPRS seems to be the best choice when the embedded system is a moving one and without a GPS, for it can get the position information through the GPRS net.

\section{Wechat adversitising value and the profit model}

The rapid development of the science and technology creates the rapid prosperity of the new media economy. In the mean time, the popularity of the mobile instant messaging application products dramatically change the way people's daily life interactions, and it quickly grows into a very popular emerging industry with a new look. Among them, Wechat, as a typical representative of the mobile Internet instant messaging product, not only brings the enormous social value and spread value, but also contains a very considerable commercial value. But now, the profit model of wechat and its similar products is not perfect, even though there are huge user groups and a vast amount of information flow, wechat and its similar products are faced with the bottlenecks of the business model innovation and marketing innovation means of communication. Therefore, in the terms of wechat or its similar products, there are important theoretical and practical significances in the research on the wechat profit model and its advertising value. Wechat QR code and marketing are shown in Fig. 3. [5]

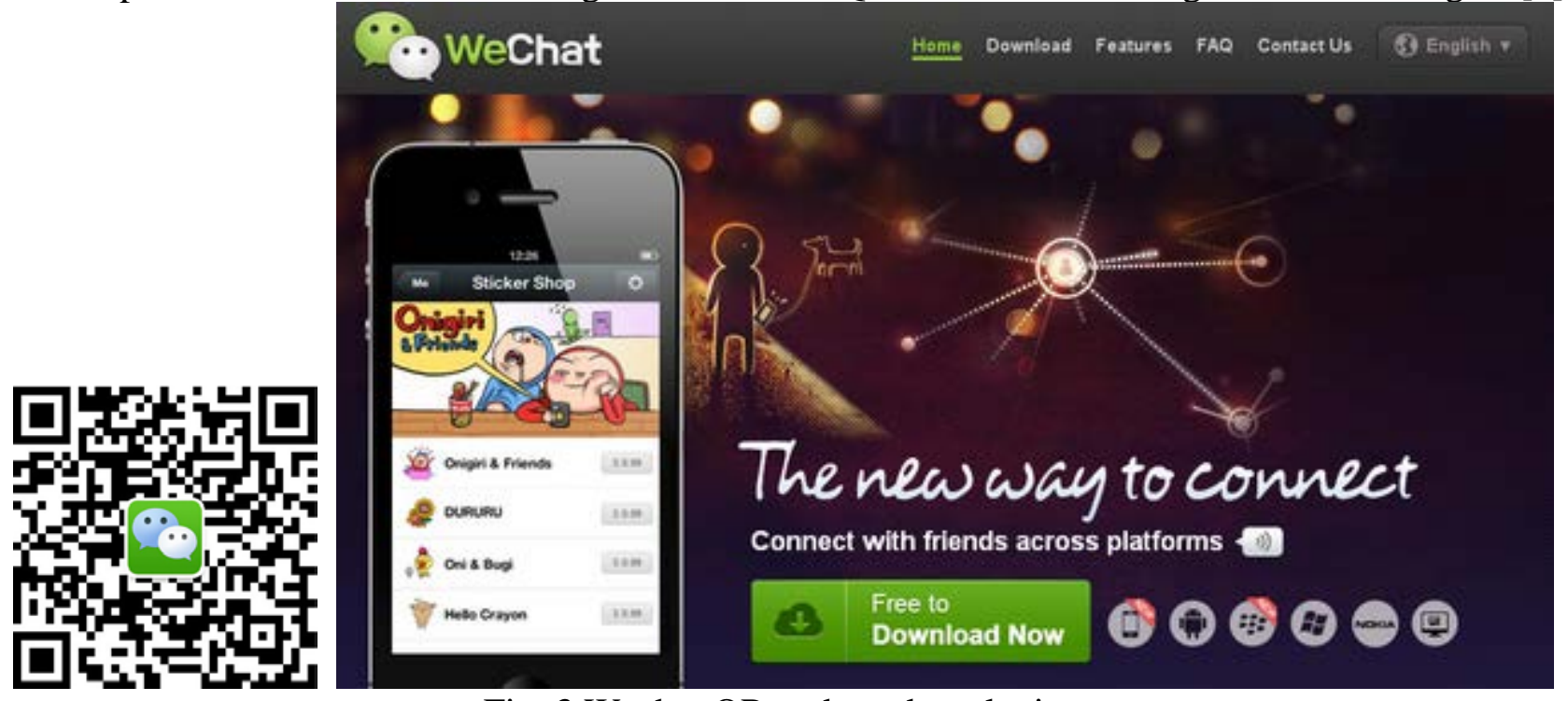

Fig. 3 Wechat QR code and marketing

The summarizes wechat and the instant messaging products industry, and lays a research background on the basis of brief description of the wechat development situation, product features and the competition of the similar products. Secondly, the analyzes the mufti-values of wechat, and analyzes the existence and development of wechat from the aspects of politic, economy and society. At the same time, we can find why wechat is profitable from the aspects of user, industry, company and product itself. Next, the study cites the Silaiwosiji profitmodel theory, and digs out the main profitability elements of wechat from the angles of profit source, profit point, profit leverage and profit barriers, and analyzes the main profit structure of wechat by drawing on successful experience of some applications which are similar to wechat. Finally, the study describes the possible enterprises marketing communication model and their own profit model now and in the future, and points out the characteristics and the revelation to the similar products, and makes the prospect of the future development trend of wechat and the similar products. A case of Wechat business is shown in Fig. 4. 


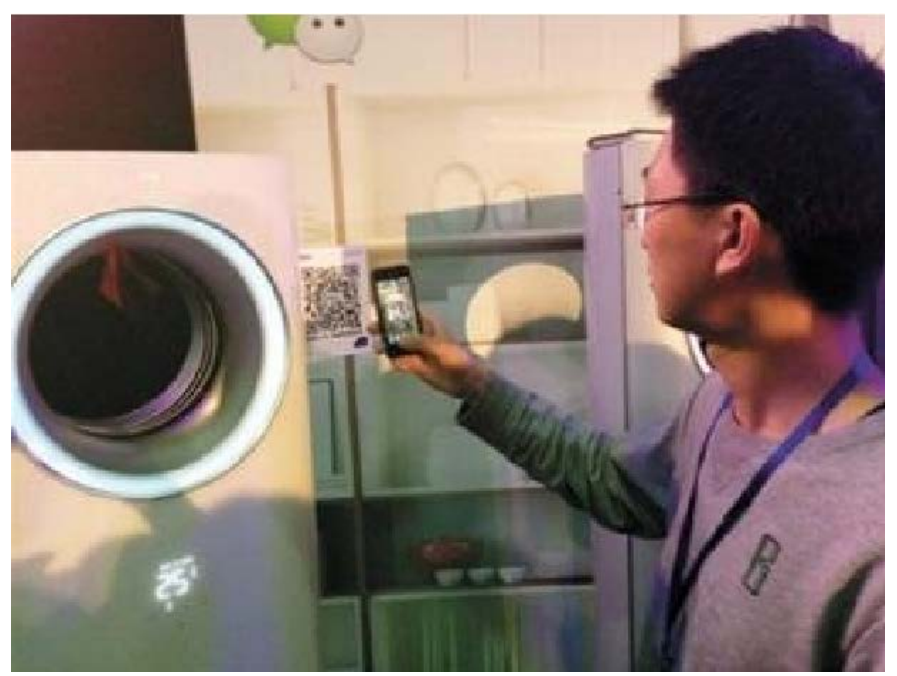

Fig. 4 Wechat business

\section{Conclusions}

In this paper, we designed and realized the system basing on Wechat public API mentioned above. Though, we have limited sensors resources and insufficient keyword database, it can deal with some normal query in natural language and return the information the users want. The showing isimperfect now that only respond in text, so in the future, we hope to update it by adding so visual representation. The development of Mobile Internet marketing in China is still at a very early stage and a big gap to the future success still exists. Developing mobile Internet marketing for small and medium-sized enterprises needs better industrial chains, as well as the cooperation and aids from governments and enterprises, so that we can build agood environment for mobile Internet marketing in the rapid and healthy development of our country. This paper conducts a preliminary study on the mobile Internet marketing issue for small and medium-sized enterprises. The results provide a reference for the further development of Mobile Internet marketing of small and medium-sized enterprises in China.

\section{References}

[1] Information on http://cn.engadget.com/2013/11/18/wechat-270-million-monthly-active-users/

[2] Kotler P. Reinventing marketing to manage the environmental imperative. Journal of Marketing, 2011, 75(4): 132-I35.

[3] Pickton D W, Wright S. What's swot in strategic analysis?. Strategic change, 2008, 7(2): 101-109.

[4] Parson V. End-to-end Internet packet dynamics. IEEE/ACM Transactions on Networking, 2007, 7(4):152.

[5] Information on http://www.wechat.com/zh_TW/ 